\title{
Path Following with Passive UHF RFID Received Signal Strength in Unknown Environments
}

\author{
Ran Liu, Artur Koch, and Andreas Zell
}

\begin{abstract}
We present a novel approach incorporating a combination of Radio-Frequency Identification (RFID) and odometry information into the motion control of a mobile robot for the purpose of path following in unknown environments. Our method utilizes RFID measurements as landmarks and makes the mobile robot autonomously follow a path that was previously recorded in a manual training phase. The approach needs no prior information about RFID sensor models, the distribution and positioning of the tags nor does it require a map of the environment. Particularly, it is adaptive to different reader power levels and various tag densities, which have a major impact on RFID performance. Extensive experiments with a SCITOS G5 robot in different environments like a library, a supermarket and hallways confirm the effectiveness of our algorithm.
\end{abstract}

\section{INTRODUCTION}

Autonomous navigation of a mobile agent along a predefined path has many applications, such as environment monitoring, service and security. Traditional methods usually use a map or landmarks to represent the environment. The robot estimates its position by matching the sensor data and the map or the landmarks, and determines its path to follow. This has been studied extensively for indoor robots with laser range finders by scan matching [1] or by comparing the similarities of visual features from camera images [2]. However, these methods require a significant amount of sensing and processing power.

Due to its simple, reliable and contactless way of identifying products, RFID has become an emerging technology and thus is already used in many industrial environments, like warehouses, stores or even libraries. Therefore, we propose a new method employing a combination of RFID and odometry measurements for path following purposes.

In particular, we apply the teaching and playback scheme to perform this task, which has already been successfully used in different navigation systems [2]. During the teaching stage, the robot is manually controlled to move along a desired path. RFID measurements and the associated motion information are recorded in an online-fashion as reference data in this phase. In the second stage, the robot shall follow this path autonomously. Therefore, we compare current RFID measurements to the previously recorded reference data to estimate the robot's relative position. As a result, motion control commands are generated by fusing the position and reference motion data to steer the robot.

R. Liu and A. Koch are with the Chair of Cognitive Systems, headed by Prof. A. Zell, Computer Science Department, University of Tübingen, Sand 1, D-72076 Tübingen, Germany \{ran.liu, artur.koch, andreas.zellduni-tuebingen.de
This work also represents an extension to our previously published approach on path following using received signal strength (RSS) only [3]. Through the integration of odometry data the robot is able to navigate along more complex paths, as opposed to following a straight route in a hallway in [3]. Odometry, on the one hand, may be used to get quite accurate estimates of the change in position over short periods of time, but on the other hand, is very sensitive to error accumulation over long distances. Therefore, we utilize a combination of RFID as well as odometry information embedded into a particle filter to compensate for the errors of both and generate a new and reliable navigation system.

The remainder of this paper is organized as follows: After an overview of the related work in Sect. II, we describe our path following method in Sect. III. Then, the details of the motion control algorithm are explained in Sect. IV. Finally, we present experimental setup and results in Sect. V, and draw conclusions in Sect. VI.

\section{RELATED WORK}

Most RFID related robotics research can be divided into sensor-model and landmark based approaches, that focus either on the mapping of the tags or on the localization of a mobile agent [4], [5], [6]. Recent research also explores the application of RFID for navigation and path following purposes. Gueaieb et al. [7] utilized the phase difference of RFID signals for the navigation task. The mobile agent was able to follow virtual paths that link the tags' orthogonal projections to the ground.

Bahl et al. [8] introduced the first fingerprinting based system (RADAR) for localization. Several distribution-based extensions have later been proposed (e.g. by Youssef et al. [9] and Ladd et al. [10]) to achieve a higher accuracy of the prior localization. Vorst et al. [11] used vector space similarity measures and weighted k-nearest neighbors (WKNN) on RFID fingerprints for the localization of the robot.

\section{ALGORITHM OVERVIEW}

\section{A. RFID Fingerprinting}

Radio frequency (RF) location fingerprinting approaches use the radio signal from RFID, WiFi access points, GSM or other RF-based sensor networks to express the observations [8]. The actual location of an agent then is estimated by matching current observations with the recorded measurements in a database. These approaches do not rely on an explicitly predicted model of the sensor's behavior. Instead, the a priori recorded set of fingerprints expresses the sensor's output at sampled locations in the global frame. 


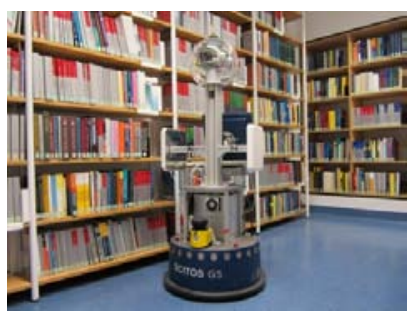

(a)

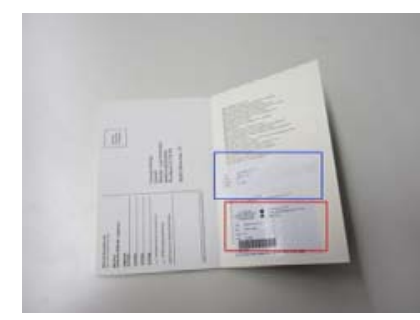

(b)
Fig. 1. (a): RFID-tagged library scenario. (b): High Frequency (HF, red) and Ultra High Frequency (UHF, blue) tags attached to the books. Only the passive UHF tags are used throughout our experiments - more information on the environment as well as the project (AmbiSense) can be obtained from http://www.ambisense.org.

Fingerprinting-based approaches therefore are assumed to be more accurate and robust in regard to location-specific distortions; for this reason, we use fingerprints in our pathfollowing approach.

\section{B. Data Recording During the Teaching Stage}

During the teaching stage, the robot is manually controlled to move along a desired path in an RFID tagged environment. While traversing the environment, arrays of RSS values $f_{t}$ of the detected tags as well as odometry obervations $m_{t}$ are gathered on the fly and stored as reference fingerprints $F_{t}$ at each time step $t: F_{t}=\left(f_{t}, m_{t}\right)$. Whereas $m_{t}=\left(v_{t}, \omega_{t}\right)$, with $v_{t}$ being the translational and $\omega_{t}$ the rotational velocities respectively. The resulting set of fingerprints $M=\left(F_{1}, F_{2}, \ldots\right)$ expresses the desired path in the spatial domain as pairs of RFID and odometry observations. Since an RFID reader may be equipped with several antennas $a \in\{1, \ldots, A\}$, each fingerprint $f_{t}$, in particular, is built up as a set of RSS observations per antenna $f_{t}=\left(f_{t}^{(1)}, \ldots, f_{t}^{(A)}\right)$. Let $L$ be the total number of tags observed by all antennas at time $t$. Then, for each antenna $a, f_{t}^{(a)}=\left(f_{t}^{(a, 1)}, \ldots, f_{t}^{(a, L)}\right)$ contains the RSS value of tag $l$ that is detected by antenna $a$ at time $t$. In our configuration, the robot has two antennas, so $A=2$. The reference fingerprints are sorted ascending over time, and each fingerprint has a unique index $i \in\{1, \ldots, I\}$, where $I$ is the total number of reference fingerprints.

\section{Navigation of the Robot in the Playback Stage}

The goal of this stage is to make the robot autonomously follow the previously defined path represented by fingerprints only. The robot's initial position, firstly, shall not be constrained to the start position of the recording, and additionally, the mobile agent shall be able to compensate for small relative offsets to the recorded path. Using odometry only, an offset to the reference path would obviously lead to bad results. Therefore we utilize RSS as well as index differences by comparing the current RFID observations with the reference fingerprints for position corrections, as detailed later (see Sect. III-E and Sect. III-F, as well as [3]). As a result, the robot can adjust its position and movement in this stage based on the RFID measurements, and we deal with the cumulative errors of the odometry.

\section{Similarity Measures}

In our approach, we individually compute the similarities between current RFID measurements and the reference data for each antenna $\operatorname{sim}\left(g_{t}^{(a)}, f_{j}^{(a)}\right)$. The overall similarity $\operatorname{sim}\left(g_{t}, f_{j}\right)$ of the current observation to a reference fingerprint is calculated by weighting the individual similarities with the number of tags detected at the respective antennas.

$$
\operatorname{sim}\left(g_{t}, f_{j}\right)=\sum_{a=1}^{A} \operatorname{sim}\left(g_{t}^{(a)}, f_{j}^{(a)}\right) \frac{n\left(g_{t}^{(a)}, f_{j}^{(a)}\right)}{\sum_{a=1}^{A} n\left(g_{t}^{(a)}, f_{j}^{(a)}\right)}
$$

Here, $n\left(g_{t}^{(a)}, f_{j}^{(a)}\right)$ is the maximum number of detected tags in $g_{t}^{(a)}$ and $f_{j}^{(a)}$ respectively. In our experiments, we use the cosine similarity (COS) which has also been utilized by Vorst et al. for localization purpose in [11].

$$
\operatorname{sim}\left(g_{t}^{(a)}, f_{j}^{(a)}\right)=\frac{\sum_{l=1}^{L} g_{t}^{(a, l)} f_{j}^{(a, l)}}{\sqrt{\sum_{l=1}^{L}\left(g_{t}^{(a, l)}\right)^{2}} \sqrt{\sum_{l=1}^{L}\left(f_{j}^{(a, l)}\right)^{2}}}
$$

\section{E. Orientation Estimation with Particle Filters}

As can be seen in Fig. 2, the angular deviation from the expected path is approximated as the index difference between the left $\left(i_{t}^{(0)}\right)$ and the right $\left(i_{t}^{(1)}\right)$ antenna:

$$
e_{t, \theta}=i_{t}^{(0)}-i_{t}^{(1)}
$$

An estimate for the index into the reference data can be calculated by a mean of the indices over both antennas:

$$
i_{t}=\frac{i_{t}^{(0)}+i_{t}^{(1)}}{2}
$$

Each antenna's index is estimated by independent particle filters. The state of each antenna is represented by $N$ samples (particles), that are updated after receiving new RFID observations. Each particle consists of a hypothesis index $x_{t}^{(n, a)}$ and a weight $w_{t}^{(n, a)}$. The estimated index of the antenna is calculated by a weighted mean over all particles:

$$
i_{t}^{(a)}=\sum_{n=1}^{N} x_{t}^{(n, a)} w_{t}^{(n, a)}
$$

Particle filters perform three central steps iteratively:

1) Prediction: The position of each particle at timestep $t$ is predicted by its previous state, a control input (e.g. odometry $m_{t}$ ) and an uncertainty/noise model:

$$
x_{t}^{(n, a)}=x_{t-1}^{(n, a)}+\mathscr{N}\left(\mu, \sigma^{2}\right)
$$

Here, $\mathscr{N}\left(\mu, \sigma^{2}\right)$ is Gaussian random noise added to the control input, with $\mu$ mean and $\sigma$ standard deviation:

$$
\mu=\frac{v_{t}}{v_{t, e}\left(K_{f}\right)} \quad \sigma=\mu \lambda
$$

In our case, the particles' positions are actually expressed in the reference fingerprints' index-frame and represented by 1D scalar values (e.g. if a particle's position is between $f_{5}$ and $\left.f_{6}, x_{t} \in[5,6]\right)$. Whereas $\sigma$ is proportional to a scalar factor $\lambda$ and the mean $\mu$, which is computed from the current velocity (based on the control input $m_{t}$ ) and the estimated reference velocity (as explained in more detail in Sect. IV). 


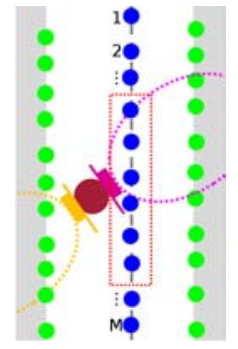

(a)

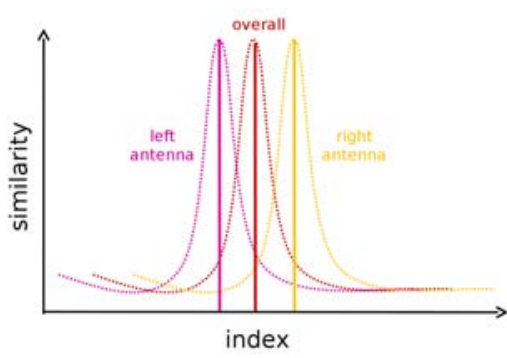

(b)
Fig. 2. Index estimation: (a) Robot following path with two antennas (orange, purple), RFID tags (green), reference fingerprints (blue) and the closest reference fingerprints (red box). (b) Respective similarities of left and right antenna, that are used to estimate the index difference as well as the overall weighted similarity.

2) Correction: The weights of the particles are updated according to the observation model $p(g \mid x, M)$, i.e., the likelihood of measurement $g_{t}$ at sample $x_{t}$ given $M$ reference fingerprints:

$$
w_{t}^{(n, a)}=\eta_{t}^{(a)} w_{t-1}^{(n, a)} p\left(g_{t}^{(a)} \mid x_{t}^{(n, a)}, M\right)
$$

Here $\eta_{t}^{(a)}$ is a normalizing factor which ensures that $\sum_{n=1}^{N} w_{t}^{(n, a)}=1$ and $M$ is the representation of the desired path (as explained in Sect. III-A).

In our method, the observation model $p\left(g_{t} \mid x_{t}^{(n, a)}, M\right)$ represents the likelihood of observing the current fingerprint $g_{t}$ from the sample position $x_{t}^{(n, a)}$ given the $M$ reference fingerprints and is computed individually for each antenna. Let $f_{j_{1}}, \ldots, f_{j_{K}}$ be the $K$ most similar reference fingerprints compared to $g_{t}$ (according to Eq. (1)). We extract those by comparing the current measurement $g_{t}$ to the reference fingerprints centered around $f_{i_{t}}$, i.e. $\left\{f_{j_{1}}, \ldots, f_{j_{K}}\right\} \in\left[f_{i_{t}-K}, f_{i_{t}+K}\right]$. This improves the robustness and reduces the computational costs if the number of the reference fingerprints is large.

$$
\begin{gathered}
p\left(g_{t}^{(a)} \mid x_{t}^{(n, a)}, m\right)= \\
\sum_{k=1}^{K} \operatorname{sim}\left(g_{t}^{(a)}, f_{j_{k}}\right) \exp \left(-\frac{1}{2} d^{2}\left(x_{t}^{(n, a)}, x_{j_{k}}\right)\right)
\end{gathered}
$$

where $d^{2}(\cdot)$ is a distance measure applied to the indices of the respective fingerprints $g$ and $f$

$$
d^{2}\left(x_{t}^{(n, a)}, x_{j_{k}}\right)=\frac{\left(x_{t}^{(n, a)}-x_{j_{k}}\right)^{2}}{\delta_{d}}
$$

and $\delta_{d}$ is the bandwidth parameter of the translational displacement component. As a result, particles close to similar reference fingerprints will get awarded by the observation model Eq. (8).

3) Resampling: If the effective sample size (ESS) falls bellow a predefined threshold, e.g. $N / 2$, resampling is performed and all degenerated particles are replaced.

\section{F. Estimation of RSS Difference}

The distance of the robot to the recorded path is expressed as the weighted average over the RSS differences between the current fingerprint and the $K$ most similar reference fingerprints.

$$
e_{t, d}=\frac{1}{\sum_{k=1}^{K} \operatorname{sim}\left(g_{t}, f_{j_{k}}\right)} \sum_{k=1}^{K} \operatorname{sim}\left(g_{t}, f_{j_{k}}\right) d_{t}\left(g_{t}, f_{j_{k}}\right)
$$

Where $d_{t}\left(g_{t}, f_{j_{k}}\right)$ is the RSS difference of the fingerprints $g_{t}$ and $f_{j_{k}}$. We assume the closest fingerprint to approximately represent the same position and therefore have similar RSS values for each detected tag. We use this difference as an approximation for the distance of the robot to the reference path. Since, in our setup (see Fig. 1(a)), the two antennas span an angle of $180^{\circ}, d_{t}\left(g_{t}, f_{j_{k}}\right)$ can be expressed as:

$$
d_{t}\left(g_{t}, f_{j_{k}}\right)=\sum_{l=1}^{L} \sum_{a=1}^{A}(-1)^{a}\left(g_{t}^{(a, l)}-f_{j_{k}}^{(a, l)}\right)
$$

\section{Control Algorithm}

Both the odometry and the RFID measurements are used for the navigation of the robot. The movement of the robot is divided into two independent components: translational $(v)$ and rotational velocity $(\omega)$. Let $K_{m}$ and $K_{f}$ be two manually set scalar parameters describing the number of the closest reference fingerprints taken for the estimation of $v$ and $\omega$, respectively. Then the estimated velocities $v_{t, e}$ and $\omega_{t, e}$ can be computed as the weighted average over the $K \in\left\{K_{m}, K_{f}\right\}$ closest reference fingerprints:

$$
\begin{gathered}
\omega_{t, e}(K)=\frac{1}{\sum_{k=i_{t}}^{i_{t}+K} \operatorname{sim}\left(g_{t}, f_{k}\right)} \sum_{k=i_{t}}^{i_{t}+K} \operatorname{sim}\left(g_{t}, f_{k}\right) \omega_{k} \\
v_{t, e}(K)=\frac{1}{\sum_{k=i_{t}}^{i_{t}+K} \operatorname{sim}\left(g_{t}, f_{k}\right)} \sum_{k=i_{t}}^{i_{t}+K} \operatorname{sim}\left(g_{t}, f_{k}\right) v_{k}
\end{gathered}
$$

The parameter $K_{m}$ is used to control the translational speed $v_{t}$. It makes the robot speed down from its current speed and thus prepare for a turn so that it can correct its position at a lower speed. The translational velocity $v_{t}$ is computed as follows:

$$
\left\{\begin{array}{lc}
v_{t}=v_{t, e}\left(K_{m}\right), & \omega_{t, e}\left(K_{m}\right)>\frac{\omega_{\max }}{K_{m}} \\
v_{t}=v_{\max }, & \text { else }
\end{array}\right.
$$

$K_{f}$ determines whether the robot should rely on RSS measurements during the navigation stage and determines the rotational speed $v_{t}$. The rotational velocity is defined as:

$$
\begin{cases}\omega_{t}=\omega_{t, e}\left(K_{f}\right), & \omega_{t, e}\left(K_{f}\right)>\frac{\omega_{\max }}{K_{f}} \\ \omega_{t}=\omega_{t, f}, & \text { else }\end{cases}
$$

$\omega_{\max }$ is the maximum rotation speed among all reference fingerprints. $\omega_{t, f}$ is the PID controller component, that combines the orientation $e_{t, \theta}$ as well as the RSS difference $e_{t, d}$ to ensure that the robot follows the reference path. Since, in our case, the RFID sample frequency is $2 \mathrm{~Hz}$ and the robot needs certain time to speed down from a higher speed, we constrain $K_{m}$ to be larger than $K_{f}$, otherwise the robot would deviate from its tracking path.

\section{EXPERIMENTS}

\section{A. Setup}

The experiments were carried out with a SCITOS G5 service robot from MetraLabs, as shown in Fig. 1. The robot is equipped with a laser range finder (SICK S300, $240^{\circ}$ field of view), and a UHF RFID reader from Impinj, Inc.. The laser scanner is used throughout our experiments to provide the ground truth. The reader is equipped with two circularly polarized antennas by Laird Technologies. 
TABLE I

DESCRIPTION OF EXPERIMENTAL DATA: RANGES (MIN.-MAX.), MEAN VALUES ( $\varnothing$ )

\begin{tabular}{|c|c|c|c|c|c|c|}
\hline Environment & $\begin{array}{c}\text { Power Level } \\
(\mathrm{dBm})\end{array}$ & $\begin{array}{c}\text { Number of RFID } \\
\text { measurements }\end{array}$ & $\begin{array}{c}\text { Trajectory length } \\
(\mathrm{m})\end{array}$ & $\begin{array}{c}\text { Duration } \\
(\mathrm{s})\end{array}$ & $\begin{array}{c}\text { Tags detected } \\
\text { per inquiry }\end{array}$ & $\begin{array}{c}\text { Number of unique } \\
\text { tags detected }\end{array}$ \\
\hline \hline Library & 30.0 & 1076 & 50.76 & 538 & $10-144, \varnothing: 50.00$ & 3313 \\
\hline Library & 27.0 & 1072 & 52.86 & 536 & $5-136, \varnothing: 33.60$ & 2397 \\
\hline Library & 24.0 & 1067 & 53.12 & 533.5 & $3-70, \varnothing: 17.52$ & 1363 \\
\hline Hallway & 30.0 & 2910 & 140.73 & 1455 & $0-210, \varnothing: 21.21$ & 725 \\
\hline
\end{tabular}

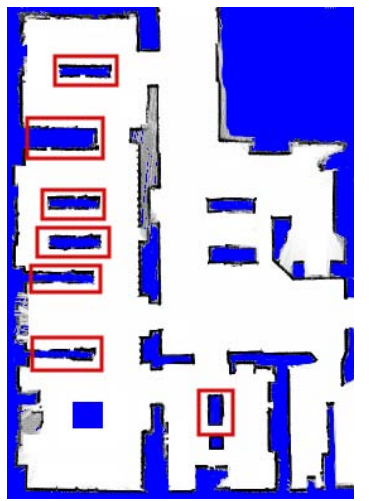

(a)

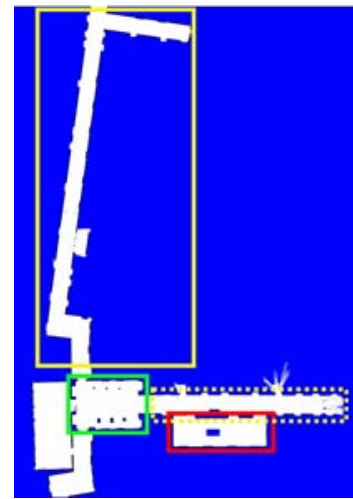

(b)
Fig. 3. The two experimental environments. (a): A library $(9 \mathrm{~m} \times 20 \mathrm{~m})$ with shelves (red boxes) of books tagged with RFID labels. (b): Hallway environment $(50 \mathrm{~m} \times 80 \mathrm{~m})$ which consists of an artificial supermarket laboratory (red box), a computer museum (green box), and corridors (orange boxes). Especially the corridor (dashed orange box) is used for performance evaluation using different parameters like varying tag densities.

It features a maximum RSS sensitivity of $-80 \mathrm{dBm}$ and a maximum read range up to approximately $7 \mathrm{~m}$.

We carried out our experiments in two kinds of environments: a library and a large indoor environment consisting of mainly hallways, as depicted in Fig. 3. Firstly, we tested our approach in a single corridor environment with varying tag densities to obtain an optimal set of parameters. The set of parameters was evaluated in another set of experiments carried out in an RFID-tagged library. In a final stage, we evaluated and verified the effectiveness and robustness of our method in a large hallway environment (Fig. 3(b)).

The library contains about 7,000 books that are labeled with passive UHF RFID tags (Alien Technology Squiggle, ISO/IEC 18000-6C), as shown in Fig. 1(b). The books are distributed on shelves at different heights reaching from $0.2 \mathrm{~m}$ to $3 \mathrm{~m}$. We additionally placed tags on the walls of the adjacent corridors (at intervals of about $0.5 \mathrm{~m}$ and different heights) to ensure an appropriate tag density in the untagged areas. For the second environment, that was partly also used by Vorst et al. in [12] (see Fig. 3(b)), we additionally placed 250 tags at the walls of the long hallway part (see solid orange box in Fig. 3(b)). These tags were distributed at different densities to verify the robustness of our algorithm under varying tag setups.

For the teaching stage, we manually steered the robot in the corridor and recorded reference log files under four kinds of tag densities, highest (23.0 tags per inquiry), high (14.7 tags per inquiry), middle (7.8 tags per inquiry) and low (6.1 tags per inquiry). The ground truth positions of the robot, computed with a laser-based Monte Carlo localization method, were recorded at the same time. For the purpose of
TABLE II

TRACKING ERRORS AND AVERAGE RUN-TIMES FOR VARYING NUMBER OF PARTICLES IN THE HALLWAY ENVIRONMENT WITH A HIGH TAG

DENSITY.

\begin{tabular}{|c|c|c|c|}
\hline $\begin{array}{c}\text { Number of } \\
\text { particles }(N)\end{array}$ & $\begin{array}{c}\text { Mean } \pm \text { std. dev. } \\
\text { Tracking Errors }(\mathrm{m})\end{array}$ & $\begin{array}{c}\text { Max. Tracking } \\
\text { Errors }(\mathrm{m})\end{array}$ & $\begin{array}{c}\text { Run-times } \\
(\mathrm{s})\end{array}$ \\
\hline 50 & $0.1094 \pm 0.0806$ & 0.3486 & 0.010 \\
100 & $0.0946 \pm 0.0530$ & 0.4412 & 0.011 \\
500 & $0.0659 \pm 0.0377$ & 0.1748 & 0.015 \\
1000 & $0.0577 \pm 0.0484$ & 0.1917 & 0.029 \\
2000 & $0.0660 \pm 0.0554$ & 0.2287 & 0.041 \\
\hline
\end{tabular}

comparing the performance of our approach under similar conditions, the recording of the references was performed on similar paths. The sample frequency of the RFID reader was set to $2 \mathrm{~Hz}$, and the maximum speed of the robot was limited to $0.1 \mathrm{~m} / \mathrm{s}$. In the library, three $\log$ files under different RFID power configurations were also recorded as reference data. As shown in Tab. I, approx. 1,100 measurements were recorded for each $\log$ file, with a distance traveled of about $50 \mathrm{~m}$ and a duration of about 10 minutes. In case of the hallway environment, the robot traveled around $140 \mathrm{~m}$ and the tag density was slightly lower.

\section{B. Impact of Particle Filter}

The influence of the number of particles $(N)$ on the tracking accuracy and the run-times of the navigation was examined in the first series of experiments. The algorithm was running on an Intel Core i5-2410M @ $2.3 \mathrm{GHz} \mathrm{CPU}$, with 4 GB RAM. As shown in Tab. II, the tracking results get worse with small $N$ (e.g., $N=50$ or 100 ). We observed that the robot could not navigate with smaller $N$ than 20. With $N>100$, we achieved nearly the same navigation accuracy. On the other hand, for larger $N$, the mean computational times increase due to the amount of particles.

In the next series of experiments, we investigated the stability of our approach for varying noise-levels $(\lambda)$ added to the odometry input, as shown in Fig. 4(a). For small $\lambda$ (less than 0.2) the results are notably worse, whereas large $\lambda$ only decrease the tracking accuracy slightly. Obviously, too small $\lambda$ values lead to an underestimation of the actual odometry error, and thus give bad results. On the other hand, if the error is assumed to be too high, the particles are spread more, and the overall particle density becomes lower. Still the estimation domain is completely sampled and covered with particles, whereas a lower particle density leads to slightly worse results due to the lower resolution. Finally, the best results regarding the experiments were achieved with $\lambda=1.0$, which seems to give the best estimation of the control input's error. 


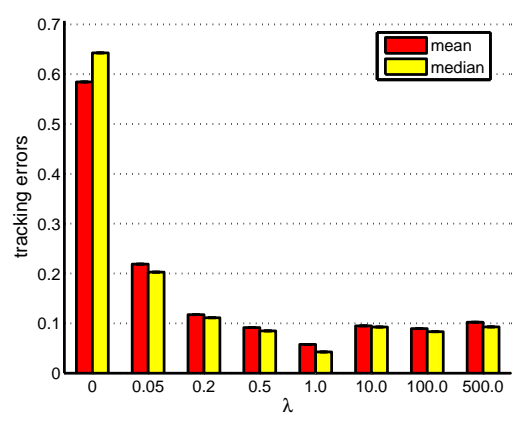

(a)

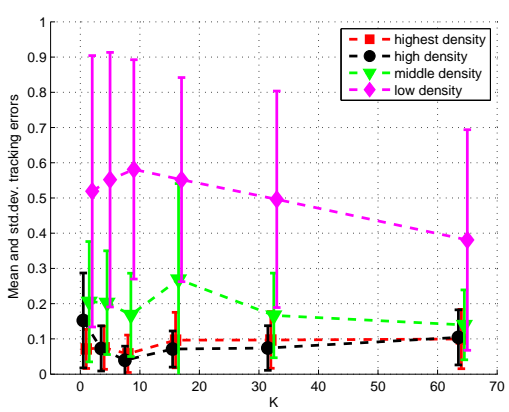

(b)

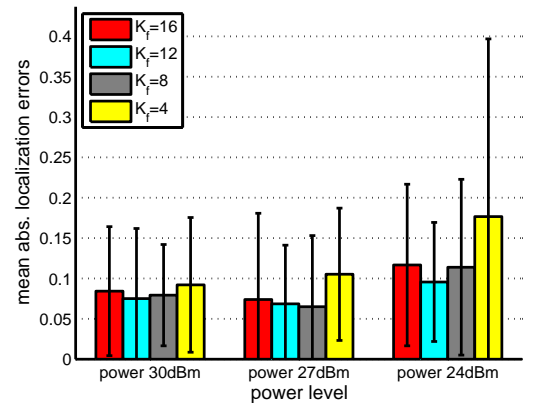

(c)

Fig. 4. Tracking accuracy under different parameters. (a): Mean and median tracking errors for varying noise $\lambda$; (b): Mean and std. dev. of tracking errors for varying tag densities; (c): Impact of different transmission power levels and $K_{f}$ on tracking errors.

\section{Influence of Different Tag Densities}

We fixed $N=1000$ and $\lambda=1$ and measured the effectiveness of our approach for various tag densities and different $K$. The experiments were carried out in a corridor in front of the supermarket laboratory. The results are shown in Fig. 4(b). As can be seen, higher tag densities lead to better results and increase the precision of our method by approx. $0.5 \mathrm{~m}$ as compared to the environment with the lowest density (for $K=8$ ). Moreover, we observed that the robot was able to track the path at a maximum speed of $0.7 \mathrm{~m} / \mathrm{s}$ for environments providing higher densities.

Generally, the choice of $K=8$ produced the best results for high tag densities. Surprisingly, this is not the case if the environment features middle and low tag densities. It is difficult to explain the direct relationship between the tracking accuracy and $K$ under middle and low tag densities. Still, an optimization of $K$ generates an enhancement in navigation precision up to $0.13 \mathrm{~m}$ and $0.2 \mathrm{~m}$ for environments with middle and low tag densities, respectively, and thus has a major impact on the tracking accuracy.

\section{Parameter Evaluation}

In the next experiments, we investigated the average speed that the robot can travel in the library under different maximum speeds $v_{\max }$ and $K_{m}$, as shown in Fig. 5. We observed that the speed and the accuracy always conflict with each other. The largest $K_{m}$ lead to the best precision, but the average speeds slightly decreased. This is because the robot spends more time to move at a low speed while adjusting its position before doing a turn. On the other hand, a smaller $K_{m}$ results in a higher average speed but higher tracking errors. In our experiments, the robot lost the path it needed to follow for $K_{m}=8$ and $v_{\max }=0.4 \mathrm{~m} / \mathrm{s}$. Yet, in the library, the turning activity happens quite often (18 times), which amounts to $70 \%$ of the whole path and thus obviously limits the overall speed of the robot. In case of large environments with less curves, the average speed would be far higher.

The hallway environment achieved a slightly lower accuracy with mean errors of $0.2 \mathrm{~m}$ (cf. Fig. 6(b)), which is due to the lower tag density. At some areas of the track, the RFID observations consisted of only one or even no tag measurements at all. As a result, the tracking accuracy

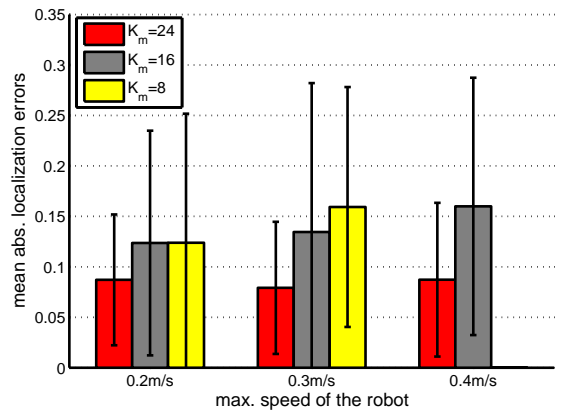

(a)

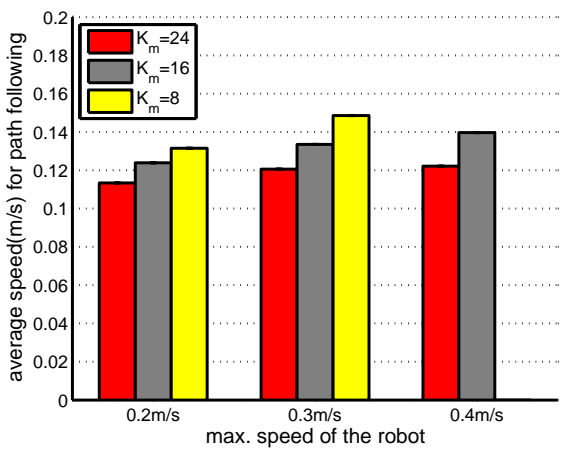

(b)

Fig. 5. Speed vs. accuracy. (a): Tracking accuracy under different maximum speeds $v_{\max }$ and $K_{m} ;(\mathrm{b})$ : Average navigation speed.

dropped to $0.6 \mathrm{~m}$, since the measured RFID readings were not sufficient to compensate for the error.

\section{E. Impact of Transmission Power}

In the next experiments, we examined the influence of $K_{f}$ under different power configurations in the library. The maximum navigation speed was set to $0.1 \mathrm{~m} / \mathrm{s}$ and the particle filter was running with a fixed amount of particles of $N=1000$. As can be seen in Fig.4(c), a too large or too small $K_{f}$ leads to higher tracking errors. The reason is, that a large $K_{f}$ gives the robot a higher belief in the odometry observations. Therefore, the robot relies too early on odometry at a turning place and thus wastes time to adjust its position through RFID observations. On the contrary, a small $K_{f}$ makes the robot trust in the RFID measurements too much and results in a delay for the robot to make a turn, since RFID observations are used to make up for the errors of the 


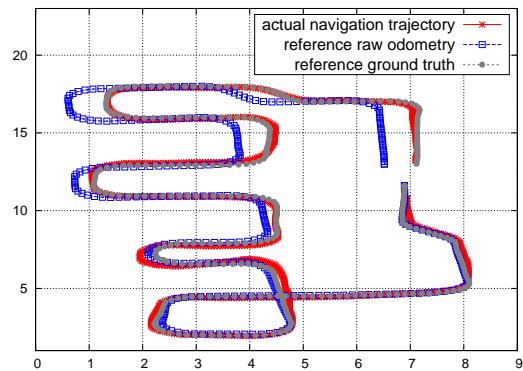

(a)

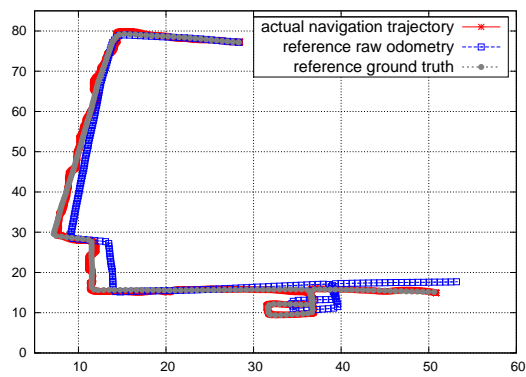

(b)

Fig. 6. Ground truth, raw odometry data, and actual trajectory. (a) Navigation results in the library: $v_{\max }=0.1 \mathrm{~m} / \mathrm{s}, K_{f}=8$, mean tracking error of $0.064 \mathrm{~m}$. (b): Navigation results in the hallway environment: $v_{\max }=0.2 \mathrm{~m} / \mathrm{s}, K_{f}=8$, mean tracking error of $0.2 \mathrm{~m}$. For a speed of $0.3 \mathrm{~m} / \mathrm{s}$ or higher, the robot lost its tracking path at areas with a low tag density.

odometry and not to make the robot turn fast. Overall, $K_{f}=8$ and $K_{f}=12$ lead to roughly the same accuracy. At full power and $K_{f}=8$, the robot achieves mean errors below $0.07 \mathrm{~m}$ with standard deviations of approx. $0.08 \mathrm{~m}$. An example of an actual trajectory under full power with $K_{f}=8$ is shown in Fig. 6. Compared with the mean error of $0.18 \mathrm{~m}$ for the raw odometry trajectory, our approach is more precise. Due to its cumulative characteristic, the localization accuracy of odometry will even get far worse for longer tracks. This can be seen in the hallway environment (Fig.6(b)), where the mean error of odometry grows to $0.9 \mathrm{~m}$.

Fig.4(c) also shows an interesting result for different transmission power levels of the RFID reader: the tracking accuracy remains the same or even improves if the power is reduced from full power $(30 \mathrm{dBm})$ to $27 \mathrm{dBm}$. But, if the power is too low $(24 \mathrm{dBm})$, the results get worse. By reducing the power, the read range becomes smaller and less tags are detected, thus the position uncertainty is lower. On the other hand, a higher transmission power will report more tags per inquiry and a larger band of RSS values. The additional observations seem to make up for the uncertainty introduced by the higher read range.

\section{CONCLUSIONS AND FUTURE WORK}

In this work, we presented a novel path following approach for mobile robots in RFID tagged environments using fused RFID and odometry fingerprints. Our method does not rely on an explicit map of the environment and is based on the teaching and playback scheme. Key parameters were evaluated in a library environment with thousands of tags. The performance of our approach was validated in a large hallway environment in regard to different setups.
At a max. speed of $0.3 \mathrm{~m} / \mathrm{s}$ and an RFID sample frequency of $2 \mathrm{~Hz}$, our method achieved mean abs. tracking errors of approx. $0.07 \mathrm{~m}$ in the library. In our current work, the max. speed of the robot is only $0.1 \mathrm{~m} / \mathrm{s}$ during the recording stage, which is slightly different to our previous work [3]. There, the robot moved along a corridor at a max. speed of $0.3 \mathrm{~m} / \mathrm{s}$ during the teaching stage. However, the tag density (about 12 tags per inquiry) was far lower than in the library, supermarket and hallway scenarios, thus the inquiry frequency was at $5 \mathrm{~Hz}$. If the reader was able to report tags in high density environments more frequently, the recording speed of the robot could be improved.

In the future, we are going to enhance the sample frequency of the RFID reader to improve the recording speed of the robot and thus minimize the efforts for the training stage. We also plan to investigate practical applications of the approach in regard to topological maps. There, paths between nodes could be used to connect distinct places through edges. A navigation from a starting node to a goal node could be achieved by traversing the appropriate edges, which would enable the robot to reach arbitrary predefined places by graph-based path planning in large-scale environments.

\section{REFERENCES}

[1] F. Lu and E. Milios, "Robot pose estimation in unknown environments by matching 2D range scans," Journal of Intelligent \& Robotic Systems, vol. 18, no. 3, pp. 249-275, 1997.

[2] L. Tang and S. Yuta, "Indoor navigation for mobile robots using memorized omni-directional images and robot's motion," in Proc. of the 2002 IEEE/RSJ Int. Conf. on Intelligent Robots and Systems (IROS 2002), Switzerland, September 2002, pp. 269-274.

[3] R. Liu, P. Vorst, A. Koch, and A. Zell, "Path following for indoor robots with RFID received signal strength," in Proc. of the 19th Int. Conf. on Software, Telecommunications and Computer Networks (SoftCOM 2011), Croatia, September 2011.

[4] D. Hähnel, W. Burgard, D. Fox, K. Fishkin, and M. Philipose, "Mapping and localization with RFID technology," in Proc. of 2004 IEEE Int. Conf. on Robotics and Automation (ICRA 2004), USA, 2004, pp. 1015-1020.

[5] P. Vorst and A. Zell, "Semi-autonomous learning of an RFID sensor model for mobile robot self-localization," in European Robotics Symposium 2008, ser. Springer Tracts in Advanced Robotics, vol. 44/2008. Springer Berlin/Heidelberg, February 2008, pp. 273-282.

[6] D. Joho, C. Plagemann, and W. Burgard, "Modeling RFID signal strength and tag detection for localization and mapping," in Proc. of 2009 IEEE Int. Conf. on Robotics and Automation (ICRA 2009), Kobe, Japan, May 2009, pp. 3160-3165.

[7] W. Gueaieb and M. Miah, "A modular cost-effective mobile robot navigation system using RFID technology," Journal of Communications, vol. 4, no. 2, pp. 89-95, 2009.

[8] P. Bahl and V. Padmanabhan, "Radar: An in-building RF-based user location and tracking system," in Proc. of the Nineteenth Annual Joint Conf. of the IEEE Computer and Communications Societies (INFOCOM 2000), vol. 2, Tel Aviv, Israel, March 2000, pp. 775-784.

[9] M. Youssef, A. Agrawala, and A. Udaya Shankar, "WLAN location determination via clustering and probability distributions," in Proc. of the IEEE Int. Conf. on Pervasive Computing and Communications (PerCom 2003), USA, March 2003, pp. 143-150.

[10] A. Ladd, K. Bekris, A. Rudys, L. Kavraki, and D. Wallach, "Roboticsbased location sensing using wireless ethernet," Wireless Networks, vol. 11, no. 1-2, pp. 189-204, 2005.

[11] P. Vorst and A. Zell, "A comparison of similarity measures for localization with passive RFID fingerprints," in Proc. of the joint Conf. of 41 st Int. Symposium on Robotics and 6th German Conf. on Robotics, Munich, Germany, June 2010, pp. 354-361.

[12] P. Vorst, S. Schneegans, B. Yang, and A. Zell, "Self-localization with RFID snapshots in densely tagged environments," in Proc. of the 2008 IEEE/RSJ Int. Conf. on Intelligent Robots and Systems (IROS 2008), Nice, France, September 2008, pp. 1353-1358. 